\title{
Effect of Unsteady Magnetic Hydrodynamic on Oscillating Flow for Fractional Burgers' Model
}

\author{
Amaal Mohi Nassief \\ Department of Mathematics, College of Science, University of Diyala, Iraq-Baghdad. \\ Corresponding Author: amalmuhi@sciences.uodiyala.edu.iq.
}

\begin{abstract}
The purpose of this paper is studying the effect of magnetic hydrodynamic (MHD) of unsteady flow with fractional Burger's model between two oscillating parallel plates. The fractional order derivative in described in the Riemann-Liouville sense. The solutions which we obtained of the velocity field and the shear stress by using Laplace transform and Fourier transform in the expression of Mittage-Lefller function. Furthermore, the influence of the parameters on the velocity field spotlighted by means of the several graphs. [DOI: 10.22401/JNUS.20.4.13]
\end{abstract}

Keywords: Fractional Derivative, Magnetic hydrodynamic, Laplace Transform, Fourier sine transform.

\section{Introduction}

The significance for flow of non-Newtonian fluids have much grown due to the development in technological capabilities (inclusive of foodstuffs, artificial propel-ants, molten plastics and numerous others), whereas, it is not easy to propose an only one model, that show characteristics of nonNewtonian fluids as in Newtonian fluids.So there were many of the constituent equations suggestion [1-3]. Rate type models have obtained particular consideration. A thermodynamic framework as being put into region to expand a rate type model referred to burger's model [4]. That applies to illustrate the movement of the earth's mantle. In addition, The Burgers' model is used to characterize visco-elastic materials, equivalent to asphalt in Geo-mechanics. Lately, Fractional derivatives [5], are discovered to particularly soft for illustrating the action of viscoelastic fluids, several researchers should investigate fully various problems associated with fluids, the constitutive equations for non-Newtonian fluids are changed by using the time derivative of an integer order called the RiemannLiouville fractional calculus operators. Najeeb. A. K et al [6] have been studied the unsteady flow of an incompressible Maxwell fluid with fractional derivative induced by a sudden drive plate, the no-slip hypothesis between the wall and the fluid is none longer solid. Constantin. $F$ et al. [7] have investigated the flow of a generalized Oldroyd -B fluid because of constantly accelerating plate, Dumitru. V.et al
[8] have studied unsteady flow of an incompressible generalized Oldroyd-B fluid induced by an infinite plate subject to a timedependent shear-stress. Dhiman. and Uma. B [9] discussed the visco-elastic flow of an incompressible generalized Oldroyd-B fluid between two infinite parallel plates. Yaqing. $\mathrm{L}$ and Liancun. $Z$ et.al [10] discussed the oscillating flows and heat transfer of a generalized Oldroyed-B fluid in a magnetic field. in Liancun. $Z$ and Xinxin. $Z$ et.al [11], discussed the MHD flow of a generalized Burgers' fluid due to an exponential accelerating plate, khan. $M$, et al [12], discussed the exact solutions for the oscillating motions of a fractional Burgers' fluid due to cosine and sine oscillations of an infinite flat plate. In Dhiman.B and Uma [13], studied the unsteady incompressible flow of a generalized Oldroyd-B fluid between two oscillating parallel plates in the presence of a transverse magnetic field.

In the present work, we studied the effect of the magnetic field on the flow of an incompressible generalized Burger's fluid. The solutions which we obtained of the velocity field and the shear stress by using Laplace transform and Fourier transform for the fractional calculus.

\section{1- Basics concepts}

In this section, we prepare some definitions which will be used. 


\section{Definition1.1 ([5], [14]) :}

The Riemann-Liouville fractional derivative of order $\sigma>0$, of function $\mathrm{X}(\mathrm{t})$ is given by

${ }_{a} D_{t}^{\sigma} X(t)=\frac{1}{\Gamma(m-\sigma)}\left(\frac{d}{d t}\right)^{m} \int_{a}^{t}(t-\delta)^{m-\sigma-1} X(\delta) d \delta$,

$m-1 \leq \sigma<m$

The initial time $a$ is often set to be zero. Where $\Gamma($.$) is the Euler gamma function$

\section{Definition1.2 ([5], [14]) :}

The Riemann-Liouville fractional integral, let $\mathrm{X}$ be an integrable function of order $\sigma>0$, define as

$$
{ }_{a} I_{t}^{\sigma} X(t)=\frac{1}{\Gamma(\sigma)} \int_{a}^{t}(t-\delta)^{\sigma-1} X(\delta) d \delta
$$

\section{Definition1.3 Generalized Mittage-leffler} function ([15-16]):

In 1971 Prabhakar introduced the three parameter Mittag-leffler function or generalized Mittage-leffler function or Prabhakar function.

$E_{a, b}^{\sigma}(x)=\sum_{m=0}^{\infty} \frac{(\sigma)_{m}}{m ! \Gamma(a m+b)} \operatorname{Re}(a>0), \operatorname{Re}(b>0)$

Where $(\sigma)_{m}=\sigma(\sigma+1) \ldots \ldots(\sigma+m-1)$

The function appeared in the kernel of a first order integrate equation which Prabhakar treated by using fractional calculus.

\section{2-The description of the problem}

Supposed that an incompressible generalized Burger's fluid limited by means of two infinite parallel plates in Fig.(1), the plates start of the beginning at relaxation at $\mathrm{t} \rightarrow 0^{+}$, the plates oscillated in its plane with the velocity $V \cos \left(w_{1} t\right)$ and $V \cos \left(w_{2} t\right)$ where $V$ is the velocity fluid, as a result shear the fluid velocity moved step by step. we have taken along for $\mathrm{x}$ - coordinate, $\mathrm{y}$ - coordinate are taken vertically to the parallel plates at sequentially in the presence of a magnetic field $\mathrm{B}_{0}$ applied parallel to $\mathrm{y}$-coordinate, hence, an initial condition and the boundary condition are given by

$$
\begin{aligned}
& u(y, 0)=0,0 \leq y \leq 1 \\
& u(d, t)=V \cos \left(w_{2} t\right), u(0, t)=V \cos \left(w_{1} t\right)
\end{aligned}
$$

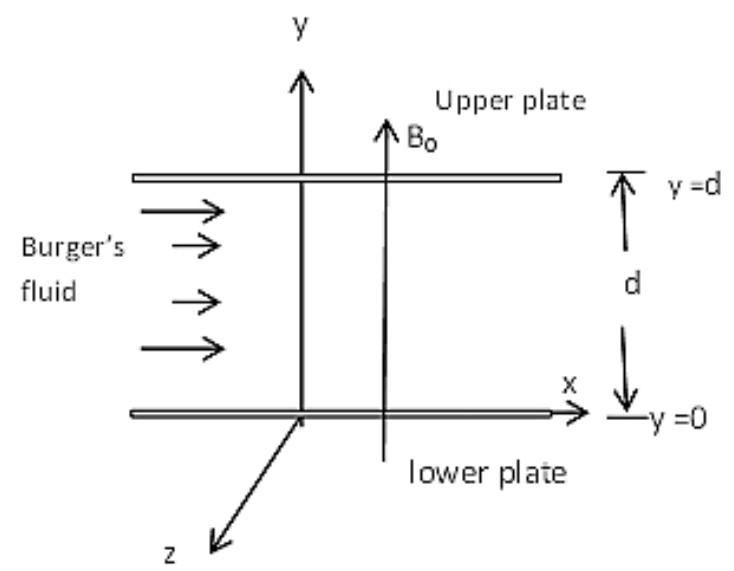

Fig.(1): Geometry model of the problem.

\section{3-Basic governing equations}

The fundamental equation of unsteady Burger's fluid and an incompressible is given by:

$$
\begin{aligned}
& T=-p I+S \\
& S\left(1+\lambda_{1}^{\alpha} \tilde{D}_{t}^{\alpha}+\lambda_{1}^{\alpha} \tilde{D}_{t}^{2 \alpha}\right)=\mu\left(1+\lambda_{3}^{\beta} \check{D}_{t}^{\beta}\right) A
\end{aligned}
$$

Where $\tilde{D}_{t}^{\alpha} s=\frac{D^{\alpha} s}{D_{t}}, \mathrm{~T}$ is the Cauchy stress tensor, -pI denotes the indeterminate spherical stress, $S$ is the extra stress tensor, $\mathrm{A}=\mathrm{L}+(\mathrm{L})^{\mathrm{T}}$ is the first Rivlin Ericksen tensor, $L=\nabla V$ is the velocity gradient, $\mu$ viscosity coefficient, $\lambda_{1}$ and $\lambda_{3}\left(<\lambda_{1}\right)$ the relaxation and retardation times respectively, $\lambda_{2}$ a new material constant of Burger's fluid and $\alpha, \beta$ are fractional parameters, such that $0 \leq \alpha \leq \beta \leq 1$ and $\check{D}_{t}^{\alpha} \quad$ converted time derivative define by

$\check{D}_{t}^{\alpha} S=D_{t}^{\alpha} S+(V . \nabla) S-L . S-S . \mathrm{L}^{T}$,

$\check{D}_{t}^{\beta} A=D_{t}^{\beta} A+L . A-L . A-A . L^{T}$

Where $\tilde{D}_{t}^{2 \alpha} S=\tilde{D}_{t}^{\alpha}\left(\tilde{D}_{t}^{\alpha} S\right)$

In the Eq.(4) and Eq.(5), $\mathrm{V}$ is the velocity vector and, $\nabla$ is the gradient operator, $D_{t}^{\alpha}, D_{t}^{\beta}$ are based on Riemann-Liouville's definition which is defined by [5],[14]as:

$D_{t}^{q} f(t)=\frac{1}{\Gamma(1-q)} \frac{d}{d t} \int_{0}^{t} \frac{f(T)}{(t-T)^{q}} d T, 0 \leq q<1$

Here $\Gamma($.$) denotes the Gamma function. We$ take the stress and velocity of the form

$S=S(y, t), V=u(y, t) \hat{\imath}$ 
Where $u(y, t)$ is the velocity component in the $\mathrm{x}$-direction, we obtain

$\nabla V=\left[\begin{array}{ccc}0 & \frac{\partial u}{\partial y} & 0 \\ 0 & 0 & 0 \\ 0 & 0 & 0\end{array}\right],(\nabla V)^{T}=\left[\begin{array}{ccc}0 & 0 & 0 \\ \frac{\partial u}{\partial y} & 0 & 0 \\ 0 & 0 & 0\end{array}\right]$

Where, $L=\nabla V$

Substituting Eq.(8) into Eq.(4), we get Eq.(9), substituting Eq.(8) into Eq.(5), we get Eq.(10), and taking account of the initial condition $S(y, 0)=0 ; \mathrm{y}>0$ the fluid begin at rest at $\mathrm{t}=0$. Where $S_{y y}=S_{z z}=S_{x z}=S_{y z}=0, S_{y x}=S_{x y}$.

$\check{D}_{t}^{\alpha} S=\left[\begin{array}{ccc}D_{t}^{\alpha} S_{x x}-2 \frac{\partial u}{\partial y} S_{x y} & 0 & 0 \\ D_{t}^{\alpha} S_{y x} & 0 & 0 \\ D_{t}^{\alpha} S_{z x} & D_{t}^{\alpha} S_{z y} & 0\end{array}\right]$

$\check{D}_{t}^{\beta} A=\left[\begin{array}{ccc}-2\left(\frac{\partial u}{\partial y}\right)^{2} & D_{t}^{\alpha} S_{y x} & 0 \\ D_{t}^{\beta}\left(\frac{\partial u}{\partial y}\right) & 0 & 0 \\ 0 & 0 & 0\end{array}\right]$

By using Eq.(9), Eq.(10) into Eq.(3), we obtain

$$
\left(1+\lambda_{1}^{\alpha} D_{t}^{\alpha}+\lambda_{2}^{\alpha} D_{t}^{2 \alpha}\right) S_{x y}=\mu\left(1+\lambda_{3}^{\beta} D_{t}^{\beta}\right) \frac{\partial u(y, t)}{\partial y}
$$

Where $S_{y x}=S_{x y}$.We consider a generalized Burger's fluid between two infinite parallel discs in a presence of a force magnetic field $B_{0}$, that acts in the direction of the positive $y$ axis, then in the presence of the magnetic body force $\varphi B_{0}^{2} u$ the equation of motion yields:

$\rho \frac{\partial u(y, t)}{\partial t}=\frac{\partial}{\partial y} S_{x y}-\varphi B_{0}^{2} u(y, t)$

Where $\varphi$ is a constant and $\rho$ is the density of fluid. Substituting $S_{x y}$ between the Eq.( 11) and Eq.(12) we have the governing equation :

$$
\begin{aligned}
& \left(1+\lambda_{1}^{\alpha} D_{t}^{\alpha}+\lambda_{2}^{\alpha} D_{t}^{2 \alpha}\right) \frac{\partial u(y, t)}{\partial t}= \\
& v\left(1+\lambda_{3}^{\beta} D_{t}^{\beta}\right) \frac{\partial^{2} u(y, t)}{\partial y^{2}}-N\left(1+\lambda_{1}^{\alpha} D_{t}^{\alpha}+\lambda_{2}^{\alpha} D_{t}^{2 \alpha}\right) u(y, t)
\end{aligned}
$$

Where $v=\frac{\mu}{\rho}$ is the kinematic viscosity and $N=\frac{\varphi B_{0}^{2}}{\rho}$

\section{4-Calculation Velocity Field:}

Manipulate the non- dimensional quantities

$u^{*}=\frac{u}{V}, y^{*}=\frac{y}{d}, t^{*}=\frac{\mu t}{p d^{2}}, \lambda_{1}^{* \alpha}=\lambda_{1}^{\alpha}\left(\frac{v}{d^{2}}\right)^{\alpha}$

$, \lambda_{2}^{* \alpha}=\lambda_{2}^{\alpha}\left(\frac{v}{d^{2}}\right)^{\alpha}, \lambda_{3}^{* \beta}=\lambda_{3}^{\beta}\left(\frac{v}{d^{2}}\right)^{\beta}, N^{*}=N \frac{d^{2}}{v^{2}}$,

$v=\frac{\mu}{\rho}$.

We obtain the Eq.(13) in non- dimensional quantities form as

$\left(1+\lambda_{1}^{\alpha} D_{t}^{\alpha}+\lambda_{2}^{\alpha} D_{t}^{2 \alpha}\right) \frac{\partial u(y, t)}{\partial t}=\left(1+\lambda_{3}^{\beta} D_{t}^{\beta}\right) \frac{\partial^{2} u(y, t)}{\partial y^{2}}$

$-N\left(1+\lambda_{1}^{\alpha} D_{t}^{\alpha}+\lambda_{2}^{\alpha} D_{t}^{2 \alpha}\right) u(y, t)$

(Omitting the dimensionless mark “*',)

$u(y, 0)=0, u(y, 0)=\cos \left(w_{1} t\right)$

$u(y, 1)=\cos \left(w_{2} t\right)$ for $t>0$,

And use finite Fourier sine transform [17] in Eq.(14), we get :

$\left(1+\lambda_{1}^{\alpha} D_{t}^{\alpha}+\lambda_{2}^{\alpha} D_{t}^{2 \alpha}\right) \frac{d}{d t} u_{s}(n, t)=$

$\left(1+\lambda_{3}^{\beta} D_{t}^{\beta}\right) \int_{0}^{1} \frac{\partial^{2} u}{\partial y^{2}} \sin (n \pi y) d y-N\left(1+\lambda_{1}^{\alpha} D_{t}^{\alpha}+\right.$

$\left.\lambda_{2}^{\alpha} D_{t}^{2 \alpha}\right) u_{s}(n, t)$

Where $u_{s}(m, t)=\int_{0}^{1} u(y, t) \sin (m \pi y) d y$ is represent the Finite Fourier sine transform of $u(y, t)$, and usage of the Eq.(16) then Eq.(17) may be rewritten as:

$$
\begin{aligned}
& \left(1+\lambda_{1}^{\alpha} D_{t}^{\alpha}+\lambda_{2}^{\alpha} D_{t}^{2 \alpha}\right) \frac{d}{d t} u_{s}(m, t)= \\
& \left(1+\lambda_{3}^{\beta} D_{t}^{\beta}\right)\left(m \pi\left((-1)^{n+1} \cos \left(w_{2} t\right)+\cos \left(w_{1} t\right)\right)-\right. \\
& \left.(m \pi)^{2} u_{s}(m, t)\right)-N\left(1+\lambda_{1}^{\alpha} D_{t}^{\alpha}+\lambda_{2}^{\alpha} D_{t}^{2 \alpha}\right) u_{s}(m, t)
\end{aligned}
$$

Applying Laplace transform to Eq. (18)[5], then using $u_{s}(m, 0)=0$, we obtain the equation

$$
\begin{aligned}
& \bar{u}_{s}(m, q)=(-1)^{m+1} \frac{m \pi q}{q^{2}+w_{2}^{2}} \\
& \frac{\left(1+\lambda_{3}^{\beta} q^{\beta}\right)}{\left((q+N)\left(1+\lambda_{1}^{\alpha} q^{\alpha}+\lambda_{2}^{\alpha} q^{2 \alpha}\right)+(m \pi)^{2}\left(1+\lambda_{3}^{\beta} q^{\beta}\right)\right)}+ \\
& \frac{m \pi q}{q^{2}+w_{1}^{2}} \frac{\left(1+\lambda_{3}^{\beta} q^{\beta}\right)}{\left((q+N)\left(1+\lambda_{1}^{\alpha} q^{\alpha}+\lambda_{2}^{\alpha} q^{2 \alpha}\right)+(m \pi)^{2}\left(1+\lambda_{3}^{\beta} q^{\beta}\right)\right)}- \\
& \frac{m \pi \lambda_{3}^{\beta}\left((-1)^{m+1}+1\right)}{\left((q+N)\left(1+\lambda_{1}^{\alpha} q^{\alpha}+\lambda_{2}^{\alpha} q^{2 \alpha}\right)+(m \pi)^{2}\left(1+\lambda_{3}^{\beta} q^{\beta}\right)\right)}
\end{aligned}
$$


We rewrite Eq. (19) into series in order to avoid the lengthy procedure of residues and integrals by using Appendix (A1)

$$
\begin{aligned}
& \overline{\mathrm{u}}_{\mathrm{s}}(\mathrm{m}, \mathrm{q})= \\
& \frac{(-1)^{\mathrm{m}+1}}{\mathrm{~m} \pi} \frac{\mathrm{q}}{\mathrm{q}^{2}+\mathrm{w}_{2}^{2}}\left(1-\sum_{\mathrm{K}=0}^{\infty} \frac{(-1)^{\mathrm{K}}}{(\mathrm{m \pi})^{2(\mathrm{~K}+1)}}\right. \\
& \sum_{\xi=0}^{\mathrm{K}+1} \frac{(\mathrm{K}+1) ! \mathrm{N}^{\xi}}{\xi !(\mathrm{K}+1-\xi) !} \sum_{\mathrm{J}=0}^{\mathrm{K}} \frac{\mathrm{K} !}{\mathrm{J} !(\mathrm{K}-\mathrm{J}) !} \sum_{\sigma=0}^{\mathrm{J}} \frac{\mathrm{J} !}{\sigma !(\mathrm{J}-\sigma) !} \\
& \left.\left(\lambda_{2}^{\alpha}\right)^{\sigma}\left(\lambda_{1}^{\alpha}\right)^{\mathrm{J}-\sigma} \frac{\mathrm{q}^{\mathrm{K}+1-\xi+\alpha \mathrm{J}+\alpha \sigma}}{\lambda_{3}^{\beta(\mathrm{K}+1)}} \frac{1+\lambda_{1}^{\alpha} \mathrm{q}^{\alpha}+\lambda_{2}^{\alpha} \mathrm{q}^{2 \alpha}}{\left(\lambda_{3}^{-\beta}+\mathrm{q}^{\beta}\right)^{\mathrm{K}+1}}\right) \\
& +\frac{1}{\mathrm{~m} \pi} \frac{\mathrm{q}}{\mathrm{q}^{2}+\mathrm{w}_{1}^{2}}(1- \\
& \sum_{\mathrm{K}=0}^{\infty} \frac{(-1)^{\mathrm{K}}}{(\mathrm{m} \pi)^{2(\mathrm{~K}+1)}} \sum_{\xi=0}^{\mathrm{K}+1} \frac{(\mathrm{K}+1) ! \mathrm{N} \xi}{\xi !(\mathrm{K}+1-\xi) !} \\
& \sum_{\mathrm{J}=0}^{\mathrm{K}} \frac{\mathrm{K} !}{\mathrm{J} !(\mathrm{K}-\mathrm{J}) !} \sum_{\sigma=0}^{\mathrm{J}} \frac{\mathrm{J} !\left(\lambda_{2}^{\alpha}\right)^{\sigma}}{\sigma !(\mathrm{J}-\sigma) ! \mathrm{n} !} \\
& \frac{1+\lambda_{1}^{\alpha} \mathrm{q}^{\alpha}+\lambda_{2}^{\alpha} \mathrm{q}^{2 \alpha}}{\left(\lambda_{3}^{-\beta}+\mathrm{q}^{\beta}\right)^{\mathrm{K}+1}}-\frac{\lambda_{3}^{\beta}}{\mathrm{m} \pi}\left((-1)^{\mathrm{m}+1}+1\right) \\
& \sum_{\mathrm{K}=0}^{\infty} \frac{(-1)^{\mathrm{K}}}{(\mathrm{m} \pi)^{2(\mathrm{~K}+1)}} \frac{1}{\lambda_{3}^{\beta(\mathrm{K}+1)}} \sum_{\mathrm{J}=0}^{\mathrm{K}} \frac{\mathrm{K} !}{\mathrm{J} !(\mathrm{K}-\mathrm{J}) !} \sum_{\omega=0}^{\mathrm{J}} \frac{\mathrm{J} !}{\omega !(\mathrm{J}-\omega) !} \\
& \sum_{\sigma=0}^{\omega} \frac{\omega !}{\sigma !(\omega-\sigma) !} \sum_{\mathrm{s}=0}^{\sigma} \frac{\sigma !}{\mathrm{s} !(\sigma-\mathrm{s}) !} \sum_{\mathrm{r}=0}^{\mathrm{s}} \frac{\mathrm{s}\left(\mathrm{N}^{\mathrm{J}-\mathrm{s}}\right)}{\mathrm{r} !(\mathrm{s}-\mathrm{r}) !}\left(\lambda_{1}^{\alpha}\right)^{\omega-\sigma+\mathrm{r}} \\
& \left(\lambda_{2}^{\alpha}\right)^{\sigma-\mathrm{r}} \mathrm{q}^{\mathrm{K}-\mathrm{J}+\mathrm{s}+\alpha(\omega+\sigma-\mathrm{r})} \frac{1}{\left(\lambda_{3}^{-\beta}+\mathrm{q}^{\beta}\right)^{\mathrm{K}+1}} \ldots \ldots \ldots . .(20)
\end{aligned}
$$

We've a major Laplace transformation of the ( $n$ - th order derivative) of Mittag-Leffler function, $E_{\alpha, \lambda}(z)$ present by [15-16]:

$$
\int_{0}^{\infty} \operatorname{Exp}(-q t) t^{b n+h-1} E_{b, \mathrm{~h}}^{(n)}\left(-a t^{b}\right) d t=\frac{n ! q^{b-h}}{\left(q^{b}+a\right)^{n+1}}
$$

\section{Where}

$E_{b, h}^{(n)}(z)=\frac{d^{n}}{d z^{n}} E_{b, h}(z)=\sum_{i=0}^{\infty} \frac{(i+n) ! z^{i}}{i ! \Gamma(b i+b n+h)}$

Taking inverse Laplace transform for Eq.(20) and by using (Appendix A3), we obtain

$u_{s}(m, t)=\frac{(-1)^{m+1}}{m \pi} \cos \left(w_{2} t\right)-\frac{(-1)^{m+1}}{m \pi}$

$\sum_{K=0}^{\infty} \frac{(-1)^{K}}{\lambda_{3}^{\beta(K+1)}(m \pi)^{2(K+1)}} \sum_{\xi=0}^{K+1} \frac{(K+1) ! N^{\xi}}{\xi !(K+1-\xi) !} \sum_{J=0}^{K} \frac{1}{J !(K-J) !}$

$\sum_{\sigma=0}^{J} \frac{J !}{(J-\sigma) ! \sigma !} \lambda_{2}^{\alpha \sigma}\left(\lambda_{1}^{\alpha(J-\sigma)}\right) \int_{0}^{t} \cos \left(w_{2}(t-\gamma)\right.$ $\left(\gamma^{\beta K+h_{1}-1} E_{\beta, h_{1}}^{(K)}\left(-\lambda_{3}^{\beta} \gamma^{\beta}\right)+\lambda_{1}^{\alpha} \gamma^{\beta k+h_{2}-1}\right.$

$\left.E_{\beta, h_{2}}^{(K)}\left(-\lambda_{3}^{\beta} \gamma^{\beta}\right)+\lambda_{2}^{\alpha} \gamma^{\beta K+h_{3}-1} E_{\beta, h_{3}}^{(K)}\left(-\lambda_{3}^{\beta} \gamma^{\beta}\right)\right) d \gamma$

$+\frac{1}{m \pi} \cos \left(w_{1} t\right)-\frac{1}{m \pi} \sum_{K=0}^{\infty} \frac{(-1)^{K}}{\lambda_{3}^{\beta(K+1)}(m \pi)^{2(K+1)}}$

$\sum_{\xi=0}^{K+1} \frac{(K+1) ! N^{\xi}}{\xi !(K+1-\xi) !} \sum_{J=0}^{K} \frac{1}{J !(K-J) !} \sum_{\sigma=0}^{J} \frac{J ! \lambda_{2}^{\alpha \sigma}}{(J-\sigma) ! \sigma !}$
$\left(\lambda_{1}^{\alpha(J-\sigma)}\right) \int_{0}^{t} \cos \left(w_{1}(t-\gamma)\left(\gamma^{\beta K+h_{1}-1} E_{\beta, h_{1}}^{(K)}\left(-\lambda_{3}^{\beta} \gamma^{\beta}\right)\right.\right.$

$+\lambda_{1}^{\alpha} \gamma^{\beta K+h_{2}-1} E_{\beta, h_{2}}^{(K)}\left(-\lambda_{3}^{\beta} \gamma^{\beta}\right)+\lambda_{2}^{\alpha} \gamma^{\beta K+h_{3}-1}$

$\left.E_{\beta, h_{3}}^{(K)}\left(-\lambda_{3}^{\beta} \gamma^{\beta}\right)\right) d \gamma-\frac{\lambda_{3}^{\beta}}{m \pi}\left(1+(-1)^{m+1}\right) \sum_{K=0}^{\infty} \frac{(-1)^{K}}{(m \pi)^{2 K}}$

$\frac{1}{\lambda_{3}^{\beta(K+1)}} \sum_{J=0}^{\mathrm{K}} \frac{1}{J !(\mathrm{K}-J) !} \sum_{\omega=0}^{J} \frac{J !}{\omega !(J-\omega) !} \sum_{\sigma=0}^{\omega} \frac{\omega !}{\sigma !(\omega-\sigma) !}$

$\sum_{s=0}^{\sigma} \frac{\sigma !}{s !(\sigma-s) !} \sum_{r=0}^{s} \frac{s !}{r !(s-r) !} N^{J-s} \lambda_{1}^{\alpha(\omega-\sigma+r)} \lambda_{2}^{\alpha(\sigma-r)}$

$\left(t^{\beta K+h_{4}-1} E_{\beta, h_{4}}^{(K)}\left(-\lambda_{3}^{\beta} t^{\beta}\right)\right)$

Where

$$
\begin{aligned}
& h_{1}=\beta-(K+1)+J-\alpha(\omega+\sigma), \\
& h_{2}=\beta-(K+1)+J-\alpha(\omega+\sigma+1), \\
& h_{3}=\beta-(K+1)+J-\alpha(\omega+\sigma+2), \\
& h_{4}=\beta-(K+1)+J+s-\alpha(\omega+\sigma-r),
\end{aligned}
$$

Utilizing inverse finite Fourier sine transform for Eq.(22), we get the velocity field

$$
\begin{aligned}
& u(y, t)=y \cos \left(w_{2} t\right)+\left(2 \sum_{m=1}^{\infty} \frac{(-1)^{m}}{m \pi} \sin (m \pi y)\right) \\
& \sum_{K=0}^{\infty} \frac{(-1)^{K}}{(m \pi)^{2(K+1)}} \frac{1}{\lambda_{3}^{\beta(K+1)}} \sum_{\xi=0}^{K+1} \frac{(K+1) !}{\xi !(K+1-\xi) !} N^{\xi} \\
& \sum_{J=0}^{K} \frac{1}{J !(K-J) !} \sum_{\sigma=0}^{J} \frac{J ! \lambda_{2}^{\alpha \sigma}}{(J-\sigma) ! \sigma !}\left(\lambda_{1}^{\alpha(J-\sigma)}\right) \int_{0}^{t} \cos \left(w_{2}(t-\gamma)\right. \\
& \left(\gamma^{\beta K+h_{1}-1} E_{\beta, h_{1}}^{(K)}\left(-\lambda_{3}^{\beta} \gamma^{\beta}\right)+\lambda_{1}^{\alpha} \gamma^{\beta k+h_{2}-1} E_{\beta, h_{2}}^{(K)}\left(-\lambda_{3}^{\beta} \gamma^{\beta}\right)\right. \\
& \left.+\lambda_{2}^{\alpha} \gamma^{\beta K+h_{3}-1} E_{\beta, h_{3}}^{(K)}\left(-\lambda_{3}^{\beta} \gamma^{\beta}\right)\right) d \gamma+(1-y) \cos \left(w_{1} t\right) \\
& -2 \sum_{m=1}^{\infty} \frac{1}{m \pi} \sin (m \pi y) \sum_{K=0}^{\infty} \frac{(-1)^{K}}{(m \pi)^{2(K+1)} \lambda_{3}^{\beta(K+1)}} \\
& \sum_{\xi=0}^{K+1} \frac{(K+1) ! N^{\xi}}{\xi !(K+1-\xi) !} \sum_{J=0}^{K} \frac{1}{J !(K-J) !} \sum_{\sigma=0}^{J} \frac{J !}{(J-\sigma) ! \cdot \sigma !} \lambda_{2}^{\alpha \sigma} \\
& \left(\lambda_{1}^{\alpha(J-\sigma)}\right) \int_{0}^{t} \cos \left(w _ { 1 } ( t - \gamma ) \left(\gamma^{\beta K+h_{1}-1} E_{\beta, h_{1}}^{(K)}\left(-\lambda_{3}^{\beta} \gamma^{\beta}\right)\right.\right. \\
& +\lambda_{1}^{\alpha} \gamma^{\beta K+h_{2}-1} E_{\beta, h_{2}}^{(K)}\left(-\lambda_{3}^{\beta} \gamma^{\beta}\right)+\lambda_{2}^{\alpha} \gamma^{\beta K+h_{3}-1} \\
& \left.E_{\beta, h_{3}}^{(K)}\left(-\lambda_{3}^{\beta} \gamma^{\beta}\right)\right) d \gamma- \\
& 2 \sum_{m=1}^{\infty} \lambda_{3}^{\beta}(1+ \\
& \left.(-1)^{m+1}\right) \sum_{s=0}^{\sigma} \frac{\sigma !}{s !(\sigma-s) !} \sum_{r=0}^{s} \frac{s !}{r !(s-r) !} N^{J-s} \\
& \lambda_{1}^{\alpha(\omega-\sigma+r)} \lambda_{2}^{\alpha(\sigma-r)}\left(t^{\beta K+h_{4}-1} E_{\beta, h_{4}}^{(K)}\left(-\lambda_{3}^{\beta} t^{\beta}\right)\right) \ldots \ldots \ldots \ldots \ldots(23)
\end{aligned}
$$

\section{5- Calculation of the shear stress:-}

Applying the Laplace transform to the equation given below:

$S_{x y}=\mu \frac{1+\lambda_{3}^{\beta} D_{t}^{\beta}}{1+\lambda_{1}^{\alpha} D_{t}^{\alpha}+\lambda_{2}^{\alpha} D_{t}^{2 \alpha}} \frac{\partial \mathrm{u}(\mathrm{y}, \mathrm{t})}{\partial \mathrm{y}}$

So that $S_{x y}=\tau(\mathrm{y}, \mathrm{t})$, hence, we obtain 
$\bar{\tau}(y, q)=\mu \frac{1+\lambda_{3}^{\beta} q_{t}^{\beta}}{1+\lambda_{1}^{\alpha} q^{\alpha}+\lambda_{2}^{\alpha} q^{2 \alpha}} \frac{\partial \overline{\mathrm{u}}(\mathrm{y}, \mathrm{q})}{\partial \mathrm{y}}$

Where $\bar{u}(y, q)$ is an image function of velocity field $u(y, t)$, therefore $\bar{u}(y, q)$ have been obtained from Eq.(23). And using the Laplace transform, we get

$\bar{u}(y, q)=y \frac{q}{q^{2}+w_{2}^{2}}+\left(2 \sum_{m=1}^{\infty} \frac{(-1)^{m}}{m \pi} \sin (m \pi y)\right)$

$\sum_{K=0}^{\infty} \frac{(-1)^{K}}{(m \pi)^{2(K+1)}} \frac{1}{\lambda_{3}^{\beta(K+1)}} \sum_{\xi=0}^{K+1} \frac{(K+1) ! N^{\xi}}{\xi !(K+1-\xi) !}$

$\sum_{J=0}^{K} \frac{1}{J !(K-J) !} \sum_{\sigma=0}^{J} \frac{J !}{(J-\sigma) ! \sigma !}\left(\lambda_{1}^{\alpha(J-\sigma)}\right) \lambda_{2}^{\alpha \sigma} \frac{\mathrm{q}}{q^{2}+w_{2}^{2}}$

$\sum_{a=0}^{\infty} \frac{\left(-\lambda_{3}^{\beta a}\right)(a+K) !}{a !}\left(\frac{1}{q^{\beta(a+K)+h_{1}}}+\lambda_{1}^{\alpha} \frac{1}{q^{\beta(a+K)+h_{2}}}+\right.$

$\left.\lambda_{2}^{\alpha} \frac{1}{q^{\beta(a+K)+h_{3}}}\right)+(1-y) \frac{\mathrm{q}}{q^{2}+w_{1}^{2}}-2 \sum_{m=1}^{\infty} \frac{1}{m \pi}$

$\sin (m \pi y) \sum_{K=0}^{\infty} \frac{(-1)^{K}}{(m \pi)^{2(K+1)} \lambda_{3}^{\beta(K+1)}} \sum_{\xi=0}^{K+1} \frac{(K+1) ! N^{\xi}}{\xi !(K+1-\xi) !}$

$\sum_{J=0}^{K} \frac{1}{J !(K-J) !} \sum_{\sigma=0}^{J} \frac{J ! \lambda_{2}^{\alpha \sigma}}{(J-\sigma) ! \sigma !}\left(\lambda_{1}^{\alpha(J-\sigma)}\right)\left(\frac{\mathrm{q}}{q^{2}+w_{2}^{2}}\right.$

$\sum_{a=0}^{\infty} \frac{(a+K) !\left(-\lambda_{3}^{\beta a}\right)}{a !}\left(\frac{1}{q^{\beta(a+K)+h_{1}}}+\lambda_{1}^{\alpha} \frac{1}{q^{\beta(a+K)+h_{2}}}\right.$

$\left.+\lambda_{2}^{\alpha} \frac{1}{q^{\beta(a+K)+h_{3}}}\right)-2 \sum_{m=1}^{\infty}\left(1+(-1)^{m+1}\right) \frac{\lambda_{3}^{\beta}}{m \pi} \sin (m \pi y)$

$\sum_{K=0}^{\infty} \frac{(-1)^{K}}{(m \pi)^{2 K} \lambda_{3}^{\beta(K+1)}} \sum_{J=0}^{\mathrm{K}} \frac{1}{J !(\mathrm{K}-J) !} \sum_{\omega=0}^{J} \frac{J !}{\omega !(J-\omega) !}$

$\sum_{\sigma=0}^{\omega} \frac{\omega !}{\sigma !(\omega-\sigma) !} \sum_{s=0}^{\sigma} \frac{\sigma !}{s !(\sigma-s) !} \sum_{r=0}^{s} \frac{s ! N-s}{r !(s-r) !} \lambda_{2}^{\alpha(\sigma-r)}$

$\lambda_{1}^{\alpha(\omega-\sigma+r)} \sum_{a=0}^{\infty} \frac{\left(-\lambda_{3}^{\beta a}\right)(a+k) !}{a !} \frac{1}{q^{\beta(a+k)+h_{4}}}$

Where, the differentiation of Eq.(25) with respect to $\mathrm{y}$, we get

$\frac{\partial u(y, q)}{\partial y}=\frac{\mathrm{q}}{q^{2}+w_{2}^{2}}+\left(2 \sum_{m=1}^{\infty}(-1)^{m} \cos (m \pi y)\right)$

$\sum_{K=0}^{\infty} \frac{(-1)^{K}}{(m \pi)^{2(K+1)}} \frac{1}{\lambda_{3}^{\beta(K+1)}} \sum_{\xi=0}^{K+1} \frac{(K+1) ! N \xi}{\xi !(K+1-\xi) !}$

$\sum_{J=0}^{K} \frac{1}{J !(K-J) !} \sum_{\sigma=0}^{J} \frac{J !}{(J-\sigma) ! \sigma !} \lambda_{2}^{\alpha \sigma}\left(\lambda_{1}^{\alpha(J-\sigma)}\right) \frac{\mathrm{q}}{q^{2}+w_{2}^{2}}$

$\sum_{a=0}^{\infty} \frac{\left(-\lambda_{3}^{\beta a}\right)(a+K) !}{a !}\left(\frac{1}{q^{\beta(a+K)+h_{1}}}+\lambda_{1}^{\alpha} \frac{1}{q^{\beta(a+K)+h_{2}}}\right.$

$\left.+\lambda_{2}^{\alpha} \frac{1}{q^{\beta(a+K)+h_{3}}}\right)+(-1) \frac{\mathrm{q}}{q^{2}+w_{1}^{2}}-2 \sum_{m=1}^{\infty} \cos (m \pi y)$

$\sum_{K=0}^{\infty} \frac{(-1)^{K}}{(m \pi)^{2(K+1)}} \frac{1}{\lambda_{3}^{\beta(K+1)}} \sum_{\xi=0}^{K+1} \frac{(K+1) !}{\xi !(K+1-\xi) !} N^{\xi}$

$\sum_{J=0}^{K} \frac{1}{J !(K-J) !} \sum_{\sigma=0}^{J} \frac{J !}{(J-\sigma) ! \sigma !} \lambda_{2}^{\alpha \sigma}\left(\lambda_{1}^{\alpha(J-\sigma)}\right)\left(\frac{\mathrm{q}}{q^{2}+w_{2}^{2}}\right.$

$\sum_{a=0}^{\infty} \frac{(a+K) !\left(-\lambda_{3}^{\beta a}\right)}{a !}\left(\frac{1}{q^{\beta(a+K)+h_{1}}}+\lambda_{1}^{\alpha} \frac{1}{q^{\beta(a+K)+h_{2}}}\right.$

$\left.+\lambda_{2}^{\alpha} \frac{1}{q^{\beta(a+K)+h_{3}}}\right)-2 \sum_{m=1}^{\infty}\left(1+(-1)^{m+1}\right) \lambda_{3}^{\beta} \cos (m \pi y)$

$\sum_{K=0}^{\infty} \frac{(-1)^{K}}{(m \pi)^{2 K} \lambda_{3}^{\beta(K+1)}} \sum_{J=0}^{K} \frac{1}{J !(K-J) !} \sum_{\omega=0}^{J} \frac{J !}{\omega !(J-\omega) !}$ $\sum_{\sigma=0}^{\omega} \frac{\omega !}{\sigma !(\omega-\sigma) !} \sum_{s=0}^{\sigma} \frac{\sigma !}{s !(\sigma-s) !} \sum_{r=0}^{s} \frac{s ! N^{J-s}}{r !(s-r) !} \lambda_{2}^{\alpha(\sigma-r)}$

$\lambda_{1}^{\alpha(\omega-\sigma+r)} \sum_{a=0}^{\infty} \frac{\left(-\lambda_{3}^{\beta a}\right)(a+k) !}{a !} \frac{1}{q^{\beta(a+k)+h_{4}}}$

Substituting the Eq.(26) into Eq.(24),we get :

$\bar{\tau}(y, q)=\frac{\mu\left(1+\lambda_{3}^{\beta} q_{t}^{\beta}\right)}{1+\lambda_{1}^{\alpha} q^{\alpha}+\lambda_{2}^{\alpha} q^{2 \alpha}}\left\{\frac{q}{q^{2}+w_{2}^{2}}+2 \sum_{m=1}^{\infty}(-1)^{m}\right.$

$\cos (m \pi y) \sum_{K=0}^{\infty} \frac{(-1)^{K}}{(m \pi)^{2(K+1)} \lambda_{3}^{\beta(K+1)}} \sum_{\xi=0}^{K+1} \frac{(K+1) ! N^{\xi}}{\xi !(K+1-\xi) !}$

$\sum_{J=0}^{K} \frac{1}{J !(K-J) !} \sum_{\sigma=0}^{J} \frac{J !}{(J-\sigma) ! \sigma !} \lambda_{2}^{\alpha \sigma}\left(\lambda_{1}^{\alpha(J-\sigma)}\right) \frac{\mathrm{q}}{q^{2}+w_{2}^{2}}$

$\sum_{a=0}^{\infty} \frac{\left(-\lambda_{3}^{\beta a}\right)(a+K) !}{a !}\left(\frac{1}{q^{\beta(a+K)+h_{1}}}+\lambda_{1}^{\alpha} \frac{1}{q^{\beta(a+K)+h_{2}}}\right.$

$\left.+\lambda_{2}^{\alpha} \frac{1}{q^{\beta(a+K)+h_{3}}}\right)+(-1) \frac{\mathrm{q}}{q^{2}+w_{1}^{2}}-2 \sum_{m=1}^{\infty} \cos (m \pi y)$

$\sum_{K=0}^{\infty} \frac{(-1)^{K}}{(m \pi)^{2(K+1)}} \frac{1}{\lambda_{3}^{\beta(K+1)}} \sum_{\xi=0}^{K+1} \frac{(K+1) !}{\xi !(K+1-\xi) !} N^{\xi}$

$\sum_{J=0}^{K} \frac{1}{J !(K-J) !} \sum_{\sigma=0}^{J} \frac{J ! \lambda_{2}^{\alpha \sigma}\left(\lambda_{1}^{\alpha(J-\sigma)}\right)}{(J-\sigma) ! \sigma !}\left(\frac{\mathrm{q}}{q^{2}+w_{2}^{2}} \sum_{a=0}^{\infty} \frac{(a+K) !}{a !}\right.$

$\left.\left(-\lambda_{3}^{\beta a}\right)\left(\frac{1}{q^{\beta(a+K)+h_{1}}}+\lambda_{1}^{\alpha} \frac{1}{q^{\beta(a+K)+h_{2}}}+\lambda_{2}^{\alpha} \frac{1}{q^{\beta(a+K)+h_{3}}}\right)\right)$

$-2 \sum_{m=1}^{\infty}\left(1+(-1)^{m+1}\right) \lambda_{3}^{\beta} \cos (m \pi y) \sum_{K=0}^{\infty} \frac{(-1)^{K}}{(m \pi)^{2 K}}$

$\frac{1}{\lambda_{3}^{\beta(K+1)}} \sum_{J=0}^{k} \frac{1}{J !(K-J) !} \sum_{J=0}^{\omega} \frac{J !}{\omega !(J-\omega) !} \sum_{\sigma=0}^{\omega} \frac{\omega !}{\sigma !(\omega-\sigma) !}$

$\sum_{s=0}^{\sigma} \frac{\sigma !}{s !(\sigma-s) !} \sum_{r=0}^{s} \frac{s !}{r !(s-r) !} N^{J-s} \lambda_{1}^{\alpha(\omega-\sigma+r)} \lambda_{2}^{\alpha(\sigma-r)}$

$\left.\sum_{a=0}^{\infty} \frac{\left(-\lambda_{3}^{\beta a}\right)(a+k) !}{a !} \frac{1}{q^{\beta(a+k)+h_{4}}}\right\}$

Lengthy but straightforward calculation allow us to determine $\tau(\mathrm{y}, \mathrm{t})$ from Eq. (28) and the simple dissolution, by using (A1, see also Appendix),we get

$$
\begin{aligned}
& \frac{1+\lambda_{3}^{\beta} q^{\beta}}{1+\lambda_{1}^{\alpha} q^{\alpha}+\lambda_{2}^{\alpha} q^{2 \alpha}}==\sum_{\partial=0}^{\infty}(-1)^{\partial} \frac{\left(\lambda_{2}^{\alpha}\right)^{\partial}}{\left(\lambda_{1}^{\alpha}\right)^{\partial+1}} q^{2 \alpha \partial} \\
& \left(\frac{q^{2 \partial \alpha}}{\left(q^{\alpha}+\frac{1}{\lambda_{1}^{\alpha}}\right)^{\partial+1}}+\lambda_{3}^{\beta} \frac{q^{2 \alpha \partial+\beta}}{\left(q^{\alpha}+\frac{1}{\lambda_{1}^{\alpha}}\right)^{\partial+1}}\right)
\end{aligned}
$$

Finally using the inverse Laplace transform for Eq.(28) to get the shear stress $\tau(\mathrm{y}, \mathrm{t})$ :

$$
\begin{aligned}
& \tau(y, t)=\mu \sum_{\partial=0}^{\infty}(-1)^{\partial} \frac{\left(\lambda_{2}^{\alpha}\right)^{\partial}}{\left(\lambda_{1}^{\alpha}\right)^{\partial+1}} \int_{0}^{t} \cos \left(w_{2}(t-\gamma)\right) \\
& \left(G_{(\alpha, 2 \alpha \partial, \partial+1)}\left(\frac{-1}{\lambda_{1}^{\alpha}}, \gamma\right)+\lambda_{3}^{\beta} G_{(\alpha, 2 \alpha \partial+\beta, \partial+1)}\left(\frac{-1}{\lambda_{1}^{\alpha}}, \gamma\right)\right) d \gamma \\
& +\left(2 \sum_{m=1}^{\infty}(-1)^{m} \cos (m \pi y)\right) \sum_{\partial=0}^{\infty}(-1)^{\partial} \sum_{K=0}^{\infty} \frac{(-1)^{K}}{(m \pi)^{2(K+1)}}
\end{aligned}
$$


$\frac{1}{\lambda_{3}^{\beta(K+1)}} \sum_{\xi=0}^{K+1} \frac{(K+1) ! N^{\xi}}{\xi !(K+1-\xi) !} \sum_{J=0}^{K} \frac{1}{J !(K-J) !} \sum_{\sigma=0}^{J} \frac{J !}{(J-\sigma) ! \sigma !}$

$\lambda_{2}^{\alpha(\sigma+\partial)} \mu\left(\lambda_{1}^{\alpha(J-\sigma-(\partial+1))}\right) \int_{0}^{t} \int_{0}^{\gamma} \cos \left(w_{2}(t-\gamma)\right) f_{K}(\delta)$

$\left(G_{(\alpha, 2 \alpha \partial, \partial+1)}\left(\frac{-1}{\lambda_{1}^{\alpha}}, \gamma-\delta\right)+\lambda_{3}^{\beta} G_{(\alpha, 2 \alpha \partial+\beta, \partial+1)}\left(\frac{-1}{\lambda_{1}^{\alpha}}, \gamma-\delta\right)\right)$

$d \delta d \gamma-\mu \sum_{\partial=0}^{\infty}(-1)^{\partial} \frac{\left(\lambda_{2}^{\alpha}\right)^{\partial}}{\left(\lambda_{1}^{\alpha}\right)^{\partial+1}} \int_{0}^{t} \cos \left(w_{1}(t-\gamma)\right)$

$\left(G_{(\alpha, 2 \alpha \partial, \partial+1)}\left(\frac{-1}{\lambda_{1}^{\alpha}}, \gamma\right)+\lambda_{3}^{\beta} G_{(\alpha, 2 \alpha \partial+\beta, \partial+1)}\left(\frac{-1}{\lambda_{1}^{\alpha}}, \gamma\right)\right) d \gamma-$

$2 \sum_{m=1}^{\infty} \cos (m \pi y) \sum_{\partial=0}^{\infty}(-1)^{\partial} \sum_{K=0}^{\infty} \frac{(-1)^{K}}{(m \pi)^{2(K+1)} \lambda_{3}^{\beta(K+1)}}$

$\sum_{\xi=0}^{K+1} \frac{(K+1) ! N}{\xi !(K+1-\xi) !} \sum_{J=0}^{K} \frac{1}{J !(K-J) !} \sum_{\sigma=0}^{J} \frac{J !}{(J-\sigma) ! \sigma !}$

$\mu \lambda_{2}^{\alpha(\sigma+\partial)}\left(\lambda_{1}^{\alpha(J-\sigma-(\partial+1))}\right) \int_{0}^{t} \int_{0}^{\gamma} \cos \left(w_{1}(t-\gamma)\right)\left(f_{K}(\delta)\right.$

$\left(G_{(\alpha, 2 \alpha \partial, \partial+1)}\left(\frac{-1}{\lambda_{1}^{\alpha}}, \gamma-\delta\right)+\lambda_{3}^{\beta} G_{(\alpha, 2 \alpha \partial+\beta, \partial+1)}\right.$

$\left.\left(\frac{-1}{\lambda_{1}^{\alpha}}, \gamma-\delta\right)\right) d \delta d \gamma-2 \mu \sum_{\partial=0}^{\infty}(-1)^{\partial} \sum_{m=1}^{\infty} \lambda_{3}^{\beta}$

$\left(1+(-1)^{m+1}\right) \cos (m \pi y) \sum_{K=0}^{\infty} \frac{(-1)^{K}}{(m \pi)^{2 K} \lambda_{3}^{\beta(K+1)}}$

$\sum_{J=0}^{K} \frac{1}{J !(K-J) !} \sum_{J=0}^{\omega} \frac{J !}{\omega !(J-\omega) !} \sum_{\sigma=0}^{\omega} \frac{\omega !}{\sigma !(\omega-\sigma) !}$

$\sum_{s=0}^{\sigma} \frac{\sigma !}{s !(\sigma-s) !} \sum_{r=0}^{s} \frac{s !}{r !(s-r) !} N^{J-s} \lambda_{1}^{\alpha(\omega-\sigma+r-(\partial+1))}$

$\sum_{a=0}^{\infty} \frac{(a+k) !}{a !}\left(-\lambda_{3}^{\beta a}\right) \int_{0}^{t} \frac{(t-\varphi)^{\beta(a+K)+h_{4}-1}}{\Gamma\left(\beta(a+K)+h_{4}\right)}$

$\left(G_{(\alpha, 2 \alpha \partial, \partial+1)}\left(\frac{-1}{\lambda_{1}^{\alpha}}, \varphi\right)+\right.$

$\left.\left.\lambda_{3}^{\beta} G_{(\alpha, 2 \alpha \partial+\beta, \partial+1)} \frac{-1}{\lambda_{1}^{\alpha}}, \varphi\right)\right) d \varphi$

Where,

$f_{K}=\sum_{a=0}^{\infty} \frac{(a+K) !}{a !}\left(-\lambda_{3}^{\beta a}\right)\left\{\frac{\delta^{\beta(a+K)+h_{1}-1}}{\Gamma\left(\beta(a+K)+h_{1}\right)}+\right.$

$\left.\lambda_{1}^{\alpha} \frac{\delta^{\beta(a+K)+h_{2}-1}}{\Gamma\left(\beta(a+K)+h_{2}\right)}+\lambda_{2}^{\alpha} \frac{\delta^{\beta(a+K)+h_{3}-1}}{\Gamma\left(\beta(a+K)+h_{3}\right)}\right\}$

\section{Results and Discussion}

In this paper, we've been offered the flow of a generalized burger's fluid between two oscillating infinite parallel plates. The velocity field and also the shear stress are determined by means that of finite Fourier sine transform and Laplace transform in series type in terms of Mittag-Leffler function. Furthermore, several shapes are sketched to show the behavior of diverse parameters contributory at the expressions of the velocity Field.

Fig.(2), show an impact of fractional parameter $\alpha$ on the movement of the fluid. The parameter $\alpha$ takes decreasing values, the velocity field will increase. Fig.(3) demonstrate the velocity changes with fractional parameter $\beta$, the velocity effect increased with increased values of $\beta$. we can see their effects on fractional parameters $\beta, \alpha$ motions are opposite. Fig.(4) shows the influence the time on the velocity field, the increasing the velocity field when $t$ increase, Fig.(5) is plotted to demonstrate the effects of magnetic hydrodynamic (MHD), It is observed that the flow velocity is a decreasing with the increasing values of the parameter N. Figs. (6) and (7) displays the influence of $w_{1}, w_{2}$ on velocity field. The velocity effect decreased with increased values of $w_{1}$. In addition we see opposite with values of $w_{2}$.

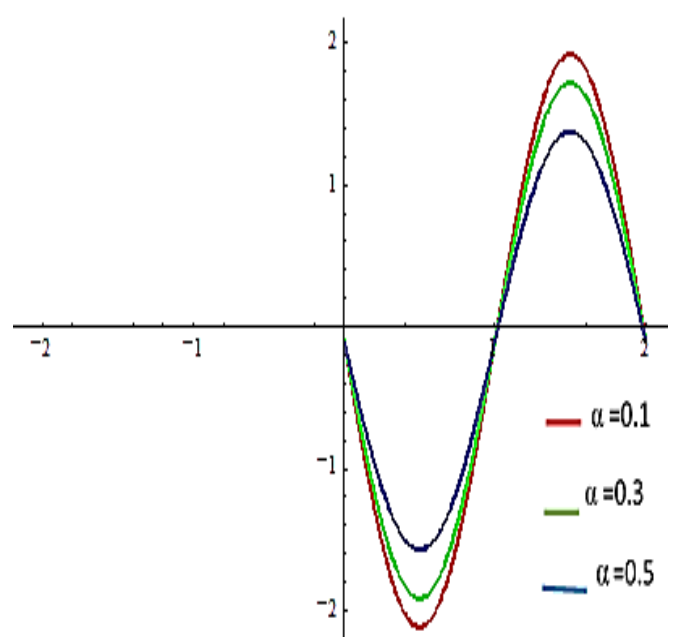

Fig.(2): Velocity Field for $\alpha=0.1,0.3,0.5$ For $N=5, \beta=0.5, t=3 \pi / 4, \lambda_{1}=3, \lambda_{2}=4, \lambda_{r}=5$, $w_{1}=1.2, w_{2}=1.4$.

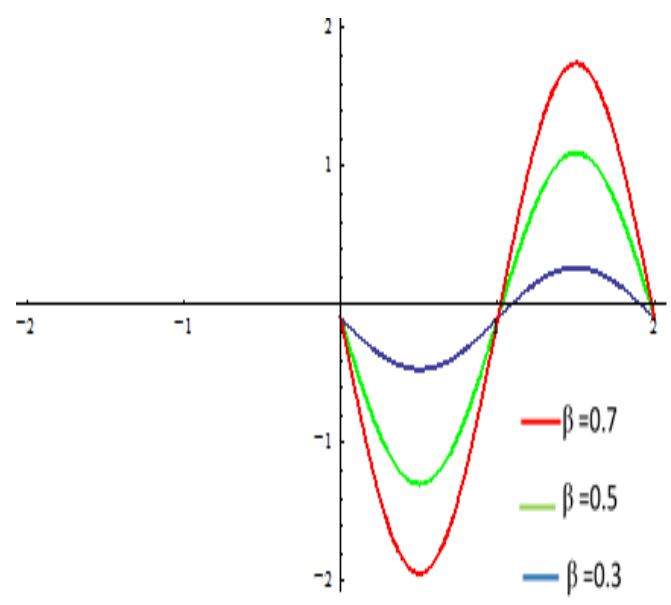

Fig.(3): Velocity Field for $\beta=0.3,0.5,0.7$ For $N=5, t=3 \pi / 4, \lambda_{1}=2, \lambda_{2}=5, \lambda_{r}=6, w_{1}=1.3$, $w_{2}=1.4, \alpha=0.5$, 


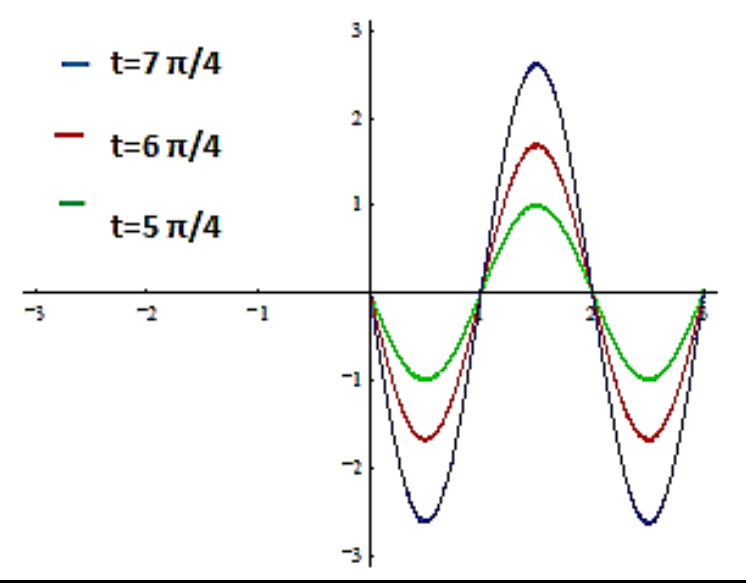

Fig.(4) : Velocity Field for $t=6 \pi / 4,5 \pi / 4,7 \pi / 4$ For $N=5, \beta=0.6, \lambda_{r}=6, \lambda_{2}=5, \lambda_{1}=3, \alpha=0.2$, $w_{1}=1.2, w_{2}=1.4$

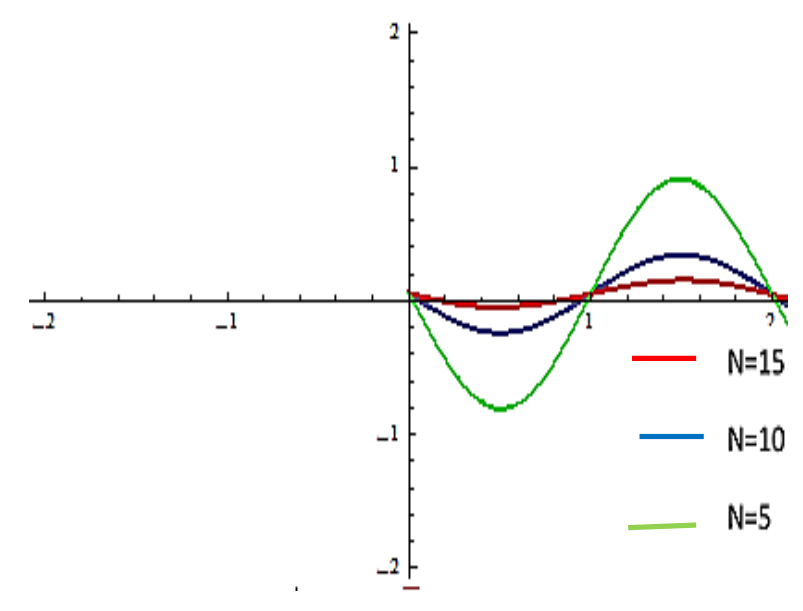

Fig. (5) : Velocity Field for $N=5,10,15$ For $t=$ $\pi / 4, \beta=0.7, \lambda_{r}=6, \lambda_{2}=4, \lambda_{1}=3, \alpha=0.3$, $w_{1}=1.2, w_{2}=1.3$.

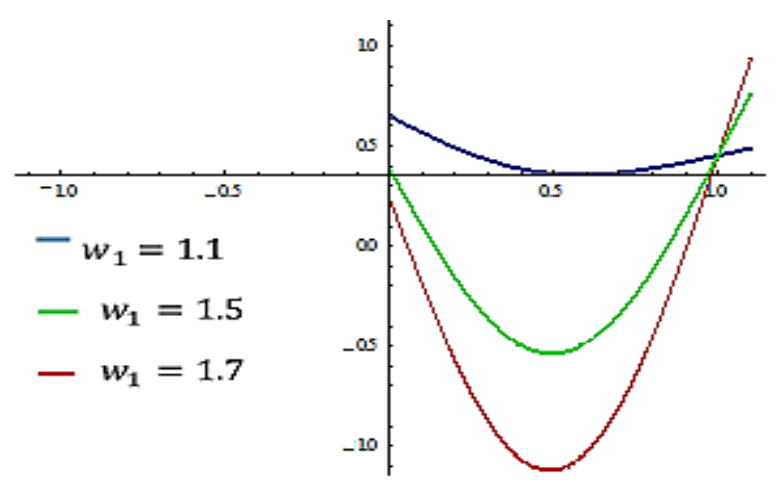

Fig.(6) : Velocity Field $w_{1}=1.1,1.5,1.7$ For $N=5, \beta=0.5, t=\pi / 4, \lambda_{r}=6, \lambda_{2}=6, \lambda_{1}=3$, $\alpha=0.6, w_{2}=1.2$.

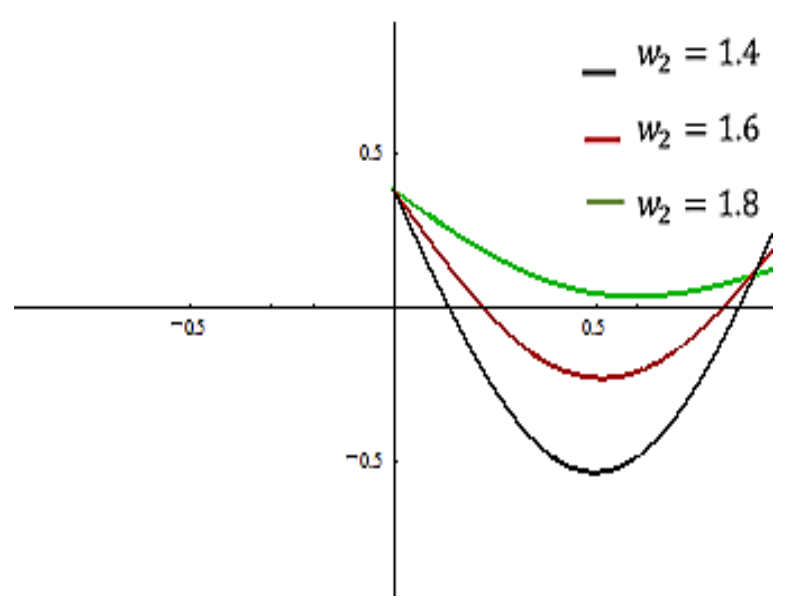

Fig.(7): Velocity Field for $w_{2}=1.4,1.6,1.8$ For $N=5, \beta=0.4, \lambda_{r}=6, \lambda_{2}=3, \lambda_{1}=2, \alpha=0.5$, $w_{1}=1.5, t=\pi / 4$

\section{Appendix:-}

(A1) $\frac{1}{z+a}=\sum_{K=0}^{\infty}(-1)^{K} \frac{z^{K}}{a^{K+1}}, \sum_{\propto=0}^{K} C_{K}^{\alpha} b^{\alpha}=$ $\sum_{\propto, l \geq 0}^{\alpha+l=K} \frac{K ! b^{\alpha}}{\alpha ! l !}$

(A2) $L^{-1}\left\{\frac{q^{b}}{\left(q^{a}-d\right)^{c}}\right\}=G_{a, b, c}(d, t)$

$\left(G_{a, b, c}(d, t)\right.$ represent a generalized $G$ - function $)$, $\operatorname{Re}(a c-b)>0, \operatorname{Re}(q)>0,\left|q^{a}\right|>|d|$

(A3) $u_{1}(t)=L^{-1}\left(u_{1}(p)\right)$ and $u_{1}(t)$

$=L^{-1}\left(u_{2}(p)\right)$ then $L^{-1}\left(u_{1}(p) u_{2}(p)\right)=\left(u_{1} * u_{2}\right)(t)$

$=\int_{0}^{t} u_{1}(t-r) u_{2}(r) d r=\int_{0}^{t} u_{1}(r) u_{2}(t-r) d r$

(A4) $\frac{t^{r}}{\Gamma(r+1)}=\frac{1}{s^{r+1}}: \operatorname{Re}(r)>-1, \operatorname{Re}(s)>0$

\section{References}

[1] Constantin. F, M. Kamran, Corina. F, Dumitru. V, "Exact solutions for the flow of a generalized Oldroyd-B fluid induced by a constantly accelerating plate between two side walls perpendicular to the plate", Journal Non-Newton Fluid Mechanics,156 (6) 189-201, (2009).

[2] Xu. M, Tan. W, "The representation of the constitutive equation of viscoelastic materials by the generalized fractional element networks and its generalized solutions", Science China Series. G, 46 (2) 145-157, (2003).

[3] Wenchang. T, Takashi. M, "Stokes' first problem for an Oldroyd-B fluid in a porous half-space", Physics of Fluids, 17(2) pp(023101-023107), (2005). 
[4] Burgers. J. M, "Mechanical Conside-ratio Model Systems Phenomenological Theories of Relaxation and of Viscosity First Report on Viscosity and Plasticity", Nordemann Publishing Company, New York, (1935).

[5] Igor. P, "Fractional differential equations", New York: Academic Press, (1999).

[6] Muhammad. J and Najeeb. A. K, "Slip Effects on Fractional Viscoelastic Fluids", International Journal of Differential Equations, Volume (2011) 2011, pp19, (2011).

[7] Dumitru. V., Constantin. F, and Corina. F, "Flow of a Generalized Oldroyd-B Fluid Due to a Constantly Accelerating Plate", Applied Mathematics and Computation, 201(1), 834-842, (2008).

[8] Dumitru. V, Constantin. F, and Corina. F, "Unsteady Flow of a Generalized OldroydB Fluid Due to an Infinite Plate Subject to a Time Dependent Shear Stress", Canadian Journal of Physics, 88, 675-687, (2010)

[9] Dhiman. B, Uma. B, "Unsteady Incompressible Flow of a Generalized Oldroyed- B Fluid between Two Infinite Parallel Plates", World Journal of Mechanics, Vol(3), 146-151, (2013).

[10] Yaqing. L, Liancun. Z, Xinxin. Z, and Fenglei. Z, "The Oscillating Flows and Heat Transfer of a Generalized Oldroyed-B Fluid in Magnetic Field", International Journal of Applied Mathematics, Vol. (40)4, (2010).

[11] Yaqing. L, Liancun. Z, Xinxin. Z, "MHD flow and heat transfer of a generalized Burgers' fluid due to an exponential accelerating plate with the effect of radiation", Computer and applying with mathematic, Vol(62), pp(3123-3131), (2011).

[12] M. Khan, A. Anjuma, C. Fetecau, H. Qi, "Exact solutions for some oscillating motions of a fractional Burgers' fluid", Mathematical and Computer Modeling. (51) pp (682-692), (2010).

[13] Dhiman. B, Uma. B, "Unsteady Incompressible Flow of a Generalized Oldroyd-B Fluid between Two Oscillating Infinite Parallel Plates in Presence of a Transverse Magnetic Field", World Journal of Mechanics, Vol. (6)1, 106-115, (2015).
[14] Almeida. R., Pooseh. S, Torres. D F. M, "Computational Methods in the Fractional Calculus of Variations", imperial college press, London, 2014.

[15] A. Erdélyi, "Higher Transcendental Functions", McGraw Hill, New York, 1955.

[16] Gorenflo. R, Kilbas. A. A. "Mainardi. F, Rogosin. S. V, "Mittag-Leffler Functions, Related Topics and Applications", springer verlag, 2014.

[17] Senddn. I. N, "Fourier Transform", McGraw Hill Company, New York, Toronto, London, 1951. 